Article

\title{
Influence of the Homogenization Pressure on the Rheology of Biopolymer-Stabilized Emulsions Formulated with Thyme Oil
}

\author{
Luis A. Trujillo-Cayado, Jenifer Santos* $*$, Nuria Calero ${ }^{(0)}$, Maria del Carmen Alfaro and \\ José Muñoz \\ Departamento de Ingeniería Química, Facultad de Química, Universidad de Sevilla, C/P. García González, 1, \\ 41012 Sevilla, Spain; ltrujillo@us.es (L.A.T.-C.); nuriacalero@us.es (N.C.); alfaro@us.es (M.d.C.A.); \\ jmunoz@us.es (J.M.) \\ * Correspondence: jsantosgarcia@us.es; Tel.: +34-954-556-447
}

Received: 14 December 2018; Accepted: 14 February 2019; Published: 18 February 2019

check for updates

\begin{abstract}
Different continuous phases formulated with ecofriendly ingredients such as AMIDET®N, an ecological surfactant, as well as welan and rhamsan gums were developed. An experimental design strategy was been in order to study the influence of the ratio of these two polysaccharides and the homogenization pressure applied in a microfluidizer on the critical shear stress for the continuous phases developed. A pure rhamsan gum solution was selected as the starting point for further study based on the production of thyme oil-in-water emulsions. The effect of the homogenization pressure on the physical stability, critical shear stress and droplet size distribution was analyzed for emulsions with optimized values of the rhamsan-welan ratio. These bioactive thyme oil-in-water emulgels could be considered as delivery systems with potential applications in the food industry.
\end{abstract}

Keywords: biopolymer; eco-friendly surfactant; microfluidization; rheology; thyme oil

\section{Introduction}

Emulsions are thermodynamically unstable and complex systems. They have several applications in many fields like pharmaceutics, the food industry, paints, agrochemistry and cosmetics due to their ability to act as drug delivery systems [1]. However, these fluids show some drawbacks because of their physical stability. Emulsions could present some destabilization mechanisms such as creaming, coalescence, flocculation and/or phase inversion. In order to extend the shelf life of emulsions, polysaccharides are added to the continuous phase. Aqueous solutions of polysaccharides possess some interesting rheological characteristics from the application point of view; i.e., viscoelastic properties and desirable flow behavior. In addition, there are several investigations that prove the essential role of polysaccharides to enhance the physical stability since they increase the continuous phase viscosity and hence reduce the movement of the droplets [2-4]. The most-used polysaccharides are xanthan and guar gum. However, there are some new ones that have recently attracted much attention, such as welan and rhamsan gums [5-7].

Both welan and rhamsan gums belong to the sphingans group. This group has a common linear tetrasaccharide backbone structure composed of glucose, glucuronic acid, rhamnose and mannose units. The difference between the gums of this group lies in the occurrence of distinct side groups. Welan gum has commercial application in the area of cement systems. It acts as a thickening, suspending, binding and emulsifying agent, as well as a stabilizer and viscosifier. On the other hand, rhamsan gum is considered a food-grade substance [8] and presents excellent suspension characteristics, even better than xanthan gum $[4,9]$. The role of rhamsan gum as a stabilizer in emulsions has been recently reported by Trujillo-Cayado et al. [5]. 
Microfluidization technology involves forcing a system pass through microchannels to a particular area by pressurizing compressed air up to about $150 \mathrm{MPa}$. It is an easy-to-use and effective method for the development of nanoemulsions [10-12]. However, this technique has been also used to modify functional properties of xanthan gum [13]. Xanthan gum can suffer ordered-disordered conformation transition using microfluidization. In addition, xanthan gum solutions present the occurrence of hydrogen bond and entanglements, which provoke the network formation [14]. Nevertheless, there are no studies about the influence of microfluidization on the properties of rhamsan or welan gums.

Lately, the use of essential oils in emulsions has increased in popularity due to their beneficial properties such as antimicrobial, antioxidant and anticancer activity. In addition, the natural characteristic of essential oils makes them very attractive to be studied as potential ingredients for emulsions [15-18]. Namely, thyme essential oil has been used to replace synthetic chemicals as food preservatives [19]. However, the major drawback of essential oils is their high tendency for oxidation and volatility.

AMIDET ${ }^{\circledR N}$, an ecological surfactant mainly containing C18 unsaturated fatty acid, is derived from renewable European rapeseed oil. This emulsifier is very interesting to be used in ecological formulations because: (i) it has no aquatic toxicity, (ii) it is easily dispersible in aqueous solutions and (iii) it has good biodegradability. Furthermore, it has been recently used in ecological matrices for cosmetics applications [20].

In this research, an experimental design strategy was been in order to study the influence of biopolymer dispersions processed by a microfluidization technique on critical shear stress. The variables selected were the rhamsan-welan gum concentration ratio and the homogenization pressure. The influence of the rhamsan-welan ratio is interesting from the applied point of view since some mixtures of gums can present synergistic or antagonistic effects $[14,21,22]$. In addition, there are no reported studies about the influence of homogenization pressure for these gum solutions. Furthermore, the effect of the homogenization pressure on the physical stability, critical shear stress and droplet size distribution were analyzed for emulgels with optimized values of the rhamsan-welan ratio. These bioactive thyme oil-in-water emulgels could be considered as active ingredient delivery systems with potential applications in the food industry.

\section{Materials and Methods}

\subsection{Materials}

Industrial grade welan gum (K1A96) and rhamsan gum (K2C401) were used as supplied by CP Kelco Company (Atlanta, GA, USA). An ecological surfactant, AMIDET®N (INCI name: PEG-4 Rapeseedamide), was provided by KAO (Tokyo, Japan). Thyme oil (Thymus vulgaris) was purchased from Sigma-Aldrich (St. Louis, MI, USA). Water used in this study was Milli-Q water (Merk Millipore, Darmstadt, Germany).

\subsection{Preparation of Samples}

A scheme of the process followed for the preparation of samples is shown in Figure 1. The surfactant $(2.5 \mathrm{wt} \%)$ was added to the biopolymers $(0.4 \mathrm{wt} \%)$ dispersion batches of $200 \mathrm{~g}$ and mixed using an IKA Eurostar for $3 \mathrm{~h}$ at $700 \mathrm{rpm}$. In the first part of the study, the continuous phase was directly passed through a Microfluidizer M110P (Microfluidics, Westwood, MA, USA) at the corresponding homogenization pressure, taking into account the design of experiments. In the second part, the development of emulsions, samples were produced by adding the oil phase ( $20 \mathrm{wt} \%)$ to the continuous phase. Following this, they were homogenized for $120 \mathrm{~s}$ at $2000 \mathrm{rpm}$ using an Ultraturrax T50 (IKA, Shanghai, China). The secondary homogenization was performed using a Microfluidizer M110P at different pressures for one pass. A total of $20 \mathrm{wt} \%$ thyme oil was chosen to obtain a non-concentrated emulsion, since essential oils are used in this type of emulsion. In addition, the concentrations of surfactant and polysaccharides were fixed, taking into account preliminary studies. 


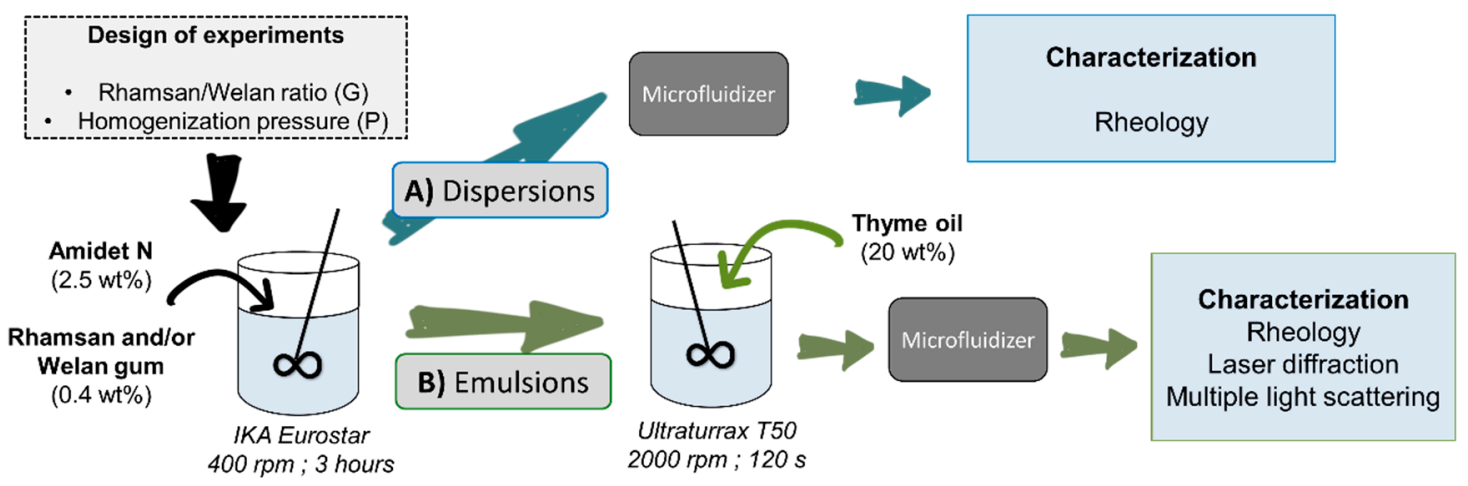

Figure 1. Scheme of the continuous phases and emulsion development.

\subsection{Rheological Characterization}

Oscillatory experiments were carried out in a controlled stress rheometer (Haake Mars II, Thermo Fisher Scientific, Waltham, MA, USA) equipped with serrated plate-plate $(\mathrm{d}=60 \mathrm{~mm})$ geometry. In order to determine the linear viscoelastic zone and critical shear stress, stress sweep tests $(0.1-20 \mathrm{~Pa})$ were conducted at a constant frequency $(0.1 \mathrm{~Hz})$. The temperature was fixed at $20^{\circ} \mathrm{C}$.

\subsection{Droplet Size Distributions by Laser Diffraction Technique}

A Malvern Mastersizer 2000 (Malvern, Worcestershire, UK) was used in order to measure the Droplet Size Distributions (DSD) for the thyme oil-in-water emulsions developed. The measurements were made in triplicate. The refraction indexes used were 1.495 and 1.33 for thyme oil and dispersed phase, respectively.

\subsection{Physical Stability of Emulsions}

In order to detect and quantify the destabilization mechanisms for the thyme oil-in-water emulsions developed, backscattering measurements (Turbiscan Lab Expert, Formulaction, Worthington, $\mathrm{OH}, \mathrm{USA}$ ) were carried out with aging time. These measurements were performed for at least $600 \mathrm{~h}$ at $25^{\circ} \mathrm{C}$. This technique, which is based in the multiple light scattering effect, has been used for different systems such as emulsions, suspensions and suspoemulsions [20,23,24]. Some authors have quantified the destabilization processes of emulsions by using the Turbiscan Stability Index (TSI) [20,25-27].

$$
T S I=\sum_{J}\left|\operatorname{scan}_{r e f}\left(h_{j}\right)-\operatorname{scan}_{i}\left(h_{j}\right)\right|
$$

where $\operatorname{scan}_{\text {ref }}$ and $\operatorname{scan}_{i}$ are the initial transmission value and the transmission value at a specific time, respectively and $h_{j}$ is a specific height in the measuring cell.

\subsection{Statistical Analysis}

In this work, design of experiments (DoE) and response surface methodology (RSM) were used to study and optimize the biopolymers formulation and production conditions. Two independent variables, the rhamsan-welan gum ratio $\left(X_{1}, G\right)$ and the homogenization pressure $\left(X_{2}, P\right)$ at five levels $(-1.414,-1,0,1$ and 1.414), were carried out. The whole design, composed of 15 experimental runs performed in random order, is shown in Table 1. Each experiment was duplicated, and the average values were used in this paper. Experimental data were fitted to a quadratic model using Echip Software.

$$
Y=\beta_{0}+\beta_{1} X_{1}+\beta_{2} X_{2}+\beta_{11} X_{1}^{2}+\beta_{22} X_{2}^{2}+\beta_{12} X_{1} X_{2}
$$

where $Y$ is the response variable, $\beta_{0}$ is a constant and $\beta_{i}$ are coefficients. For model construction, terms with $p>0.05$ were removed. 
Table 1. Experimental design carried out for the continuous phases studied. $X_{1}=$ rhamsan-welan ratio. $\mathrm{X}_{2}=$ homogenization pressure. (Rhamsan-welan ratio $=0$ means $100 \mathrm{wt} \%$ of welan gum and 1 means $100 \mathrm{wt} \%$ of rhamsan gum).

\begin{tabular}{ccccc}
\hline Sample & $\mathbf{X}_{\mathbf{1}}$ & $\mathbf{X}_{\mathbf{2}}$ & Rhamsan-Welan Ratio & Pressure (bar) \\
\hline $\mathbf{1}$ & -1 & -1 & 0.3 & 100 \\
$\mathbf{2}$ & 1 & -1 & 0.7 & 100 \\
$\mathbf{3}$ & -1 & 1 & 0.3 & 600 \\
$\mathbf{4}$ & 1 & 1 & 0.7 & 600 \\
$\mathbf{5}$ & 0 & 0 & 0.5 & 350 \\
$\mathbf{6}$ & 0 & 0 & 0.5 & 350 \\
$\mathbf{7}$ & 0 & 0 & 0.5 & 350 \\
$\mathbf{8}$ & -1.414 & 0 & 0 & 350 \\
$\mathbf{9}$ & -1 & 0 & 0.3 & 350 \\
$\mathbf{1 0}$ & 1 & 0 & 0.7 & 350 \\
$\mathbf{1 1}$ & 1.414 & 0 & 1 & 350 \\
$\mathbf{1 2}$ & 0 & -1.414 & 0.5 & 0 \\
$\mathbf{1 3}$ & 0 & -1 & 0.5 & 100 \\
$\mathbf{1 4}$ & 0 & 1 & 0.5 & 600 \\
$\mathbf{1 5}$ & 0 & 1.414 & 0.5 & 700 \\
\hline
\end{tabular}

\section{Results and Discussion}

\subsection{Development of a Continuous Phase Containing Rhamsan and/or Welan Gums}

The viscoelastic properties of the biopolymers used were determined via oscillatory shear tests. Oscillatory shear tests, and more specifically stress sweeps, can be used to analyze the structure of biopolymers. In a stress sweep, it is possible to distinguish two different zones, namely a linear viscoelastic region (LVR), in which the storage modulus $\left(G^{\prime}\right)$ and the loss modulus $\left(G^{\prime \prime}\right)$ are constant up to a critical shear stress $\left(\sigma_{\mathrm{c}}\right)$, and a non-linear one where $\mathrm{G}^{\prime}$ and $\mathrm{G}^{\prime \prime}$ start to diminish. In the LVR, which is defined as the stress and strain amplitudes ranges where $G^{\prime}$ as well as $G^{\prime \prime}$ remain independent of the applied stress and strain, the deformation in the material structure is reversible. Therefore, the amplitude of the linear viscoelastic range and the value of the critical stress can be utilized to describe polymer structure strength. Stress sweeps were performed as a function of the rhamsan-welan ratio $\left(X_{1}\right)$ and the homogenization pressure $\left(X_{2}\right)$ at $0.1 \mathrm{~Hz}$. From Figures 2 and 3 , it is possible to distinguish the two distinct zones, namely the LVR $\left(\sigma<\sigma_{\mathrm{c}}\right)$ and the non-linear zone $\left(\sigma>\sigma_{\mathrm{c}}\right)$. With increasing stress and after passing the critical shear stress, $G^{\prime}$ and $G^{\prime \prime}$ begin to decrease sharply due to the deformation of the gum samples. The effect of the rhamsan-welan gum ratio on the viscoelastic properties was studied, and the results are shown in Figure 2 and Table 2. The rhamsan-welan ratio was set at 0 , $0.3,0.5,0.7$ and 1 (where 0 means $100 \mathrm{wt} \%$ of welan gum and 1 means $100 \mathrm{wt} \%$ of rhamsan gum), while the homogenization pressure was fixed at 350 bar. In all tested samples as a function of the rhamsan-welan gum ratio (see Figure 2), the loss modulus was greater than the storage modulus in the linear viscoelastic range, as the viscous component dominates the elastic component. This fact may be due to the destruction of the three-dimensional structure of the biopolymer solution due to its introduction into the homogenizer at high pressures $(P=350 \mathrm{bar})$. In addition, it was observed that there was a tendency of higher values of the critical shear stress defining the LVR as the concentration of welan gum decreased and the rhamsan gum concentration increased. This fact can be verified by the comparison of the values of the critical shear stress of samples 5, 6, 7, 8, 9, 10 and 11 in Table 2 and in the inset of Figure 2. Therefore, it can be concluded that increasing the rhamsan gum concentration led to increase in the structural strength of samples and made it more rigid. The critical shear stress, storage modulus and loss modulus increased with the rhamsan gum concentration, which may be associated with the ability of this biopolymer to form stronger structures. This fact reveals that rhamsan gum solutions may possess a higher number of molecular aggregates through hydrogen bonds and polymer entanglement than welan gum solutions, similarly to xanthan gum solutions [14]. The use of welan 
and rhamsan gum in identical concentrations (samples 5, 6 and 7) did not lead to a positive synergistic effect on the stress sweeps, which was shown by other mixtures of gums [22]. This means that the resulting dispersion did not show more marked elastic properties. The influence of the homogenization pressure on the $G^{\prime}, G^{\prime \prime}$ and $\sigma_{\mathrm{c}}$ is illustrated in Figure 3. To determine the effect of the homogenization pressure $(P)$ on the viscoelastic properties, the rhamsan-welan gum ratio was $0.5(50 \mathrm{~W} / 50 \mathrm{R})$ while $P$ was controlled at $0,100,350,600$ and 700 bar. It was observed that when the homogenization pressure increased, the critical shear stress values decreased (inset Figure 3), showing weaker structures. In addition, it is obviously shown in Figure 3 that the magnitudes of loss and storage moduli values at the LVR decreased with an increase in homogenization pressure, thus resulting in deteriorating structural strength of biopolymer solutions. Furthermore, the storage modulus was greater than the loss modulus in the linear viscoelastic range for homogenization pressures equal to or below 100 bar. The abovementioned entanglements could break via the application of the homogenization pressure by the microfluidization technique. This fact has been previously reported in xanthan gum solutions $[13,14]$.

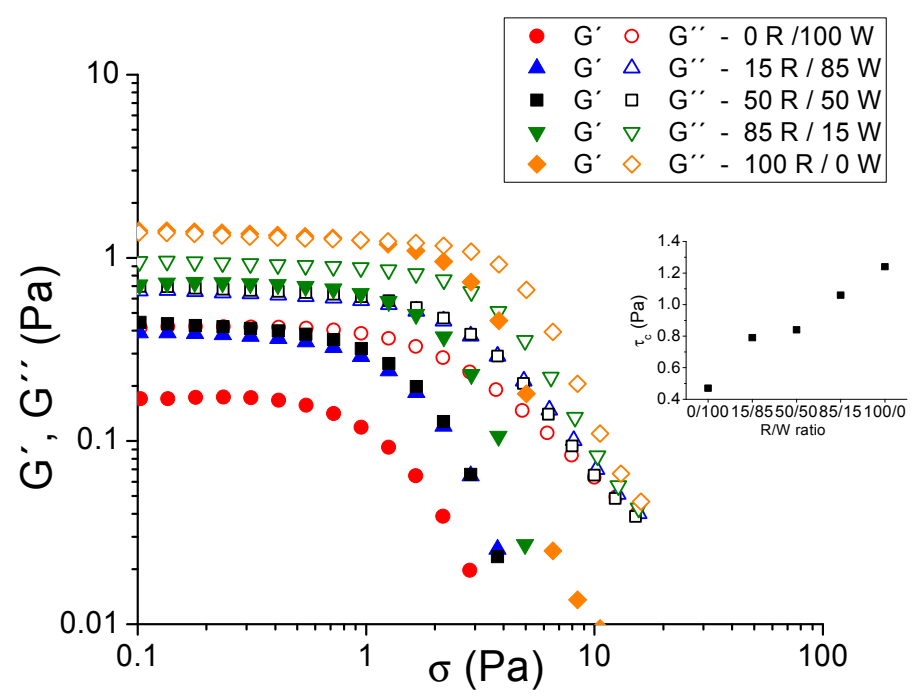

Figure 2. Stress sweeps for the continuous phases passed through a microfluidizer at 350 bar as a function of the rhamsan-welan ratio at $0.1 \mathrm{~Hz}$.

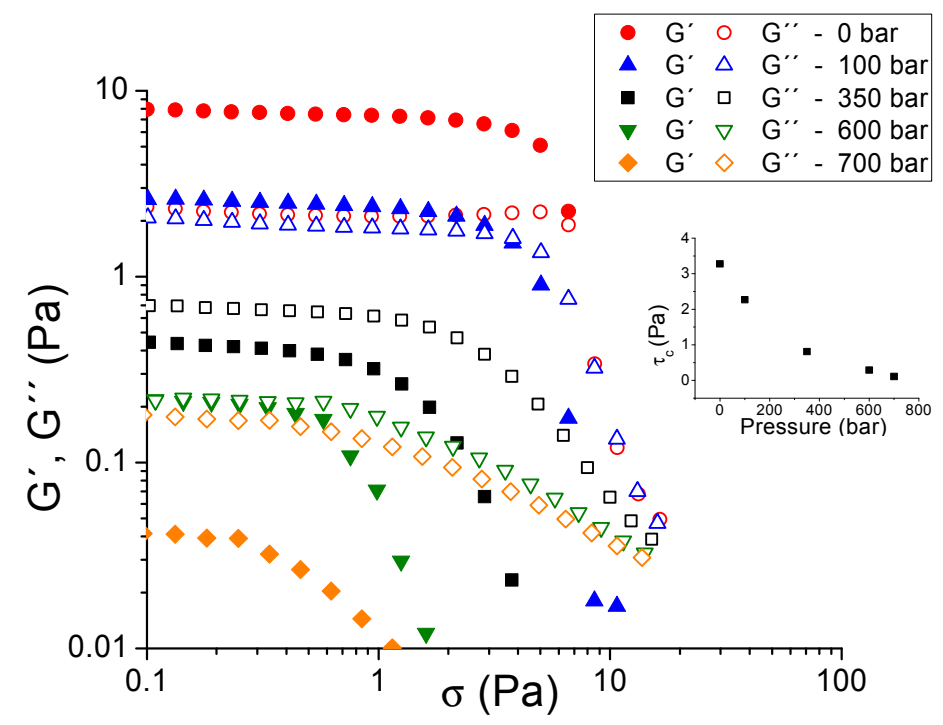

Figure 3. Stress sweeps for the continuous phase studied (Rhamsan-Welan ratio 0.5) as a function of the homogenization pressure applied in a microfluidizer at $0.1 \mathrm{~Hz}$. 
Table 2. Critical shear stress obtained from stress sweeps for the continuous phases studied.

\begin{tabular}{cccc}
\hline Sample & Rhamsan-Welan Ratio & Pressure (bar) & $\boldsymbol{\tau}_{\mathbf{c}}(\mathbf{P a})$ \\
\hline $\mathbf{1}$ & 0.3 & 100 & $1.45 \pm 0.09$ \\
$\mathbf{2}$ & 0.7 & 100 & $2.80 \pm 0.14$ \\
$\mathbf{3}$ & 0.3 & 600 & $0.13 \pm 0.01$ \\
$\mathbf{4}$ & 0.7 & 600 & $0.15 \pm 0.01$ \\
$\mathbf{5}$ & 0.5 & 350 & $0.83 \pm 0.05$ \\
$\mathbf{6}$ & 0.5 & 350 & $0.81 \pm 0.06$ \\
$\mathbf{7}$ & 0.5 & 350 & $0.84 \pm 0.05$ \\
$\mathbf{8}$ & 0 & 350 & $0.47 \pm 0.02$ \\
$\mathbf{9}$ & 0.3 & 350 & $0.79 \pm 0.04$ \\
$\mathbf{1 0}$ & 0.7 & 350 & $1.06 \pm 0.08$ \\
$\mathbf{1 1}$ & 1 & 350 & $1.24 \pm 0.08$ \\
$\mathbf{1 2}$ & 0.5 & 0 & $3.28 \pm 0.19$ \\
$\mathbf{1 3}$ & 0.5 & 100 & $2.27 \pm 0.12$ \\
$\mathbf{1 4}$ & 0.5 & 600 & $0.29 \pm 0.02$ \\
$\mathbf{1 5}$ & 0.5 & 700 & $0.11 \pm 0.01$ \\
\hline
\end{tabular}

The design of experiment (DoE) and response surface methodology (RSM) allow the evaluation of multiple interactions between independent variables in experiments. The analysis of the interactions between the homogenization pressure $\left(X_{2}\right)$ and gums ratio $\left(X_{1}\right)$ and associated output response (critical shear stress values), instead of the conventional one-factor-at-a-time method, allows a better understanding of the influence of these input factors on the structure of biopolymer solutions. This can be observed in Table 2, where critical shear stress ranges from $0.13 \pm 0.01$ to $3.28 \pm 0.19 \mathrm{~Pa}$. Moreover, a quadratic model was performed to represent the critical stress as a function of the ratio of the gums and the homogenization pressure in the chosen ranges, which is written according to the following equation $\left(R^{2}=0.988\right)$ :

$$
\sigma_{C}=0.89+0.28 \cdot X_{1}-1.06 \cdot X_{2}-0.33 \cdot X_{1} \cdot X_{2}+0.38 \cdot X_{2}^{2} .
$$

Interestingly, the critical stress was sensitive to all studied variables. In fact, $\sigma_{\mathrm{c}}$ increased with the rhamsan gum concentration (lower rhamsan-welan ratio, $X_{1}$ ) as indicated by the comparison of systems $8,9,5 / 6 / 7,10$ and 11 . This fact proves important in the selection of an adequate biopolymer as a viscosity modifier for emulsions since it also modifies the continuous phase viscosity. In contrast, the rise of the homogenization pressure $\left(X_{2}\right)$ yielded the most significant effect as supported by their respective linear and quadratic coefficients values, since the critical stress decreased from $3.28 \pm 0.19$ to $0.11 \pm 0.01$ as the pressure increased from 0 to 700 bar. The contribution of the squared term coefficient of $X_{2}$ must be also taken into consideration. Finally, it an interaction between both factors, $X_{1}$ and $X_{2}$, was observed. This fact may indicate that the gums have different behaviors against the application of the homogenization pressure by means of the microfluidizer. Since the coefficient is negative, a higher concentration of welan gum (lower value of $X_{1}$ ) and an increase of homogenization pressures (higher value of $\mathrm{X}_{2}$ ) reduce the critical stress. Thus, this biopolymer exhibits less resistance against the pressure applied, reducing to a greater extent the strength of its structure.

Figure 4 illustrates the three-dimensional response surface curve of critical shear stress $\left(\sigma_{\mathrm{c}}\right)$ for the rhamsan-welan gum concentration ratio and homogenization pressure. This figure is the representation of Equation (3), which relates the critical shear stress with the variables studied. An optimum formulation and homogenization pressure can be set for obtaining a biopolymer dispersion with enhanced critical shear stress. According to the surface response analysis, the maximum critical shear stress was obtained for $X_{1}=0$ (rhamsan-welan ratio $=0.5$ ) and $X_{2}=-1.41$ $(P=0$ bar). However, taking into account Equation (3), the predicted and extrapolated maximum critical shear stress was obtained when $X_{1}=1.414$ (only rhamsan gum) and $X_{2}=0$ ( 0 bar). In order to produce emulsions with minimum droplet sizes and enhanced physical stability, the control of 
the formulation and processing conditions is required. As a result of these preliminary tests, it was suggested that the use of pure rhamsan gum solutions allows the production of continuous phases with higher critical shear stresses. For this reason, this ratio (100 rhamsan-0 welan) was fixed for further study.

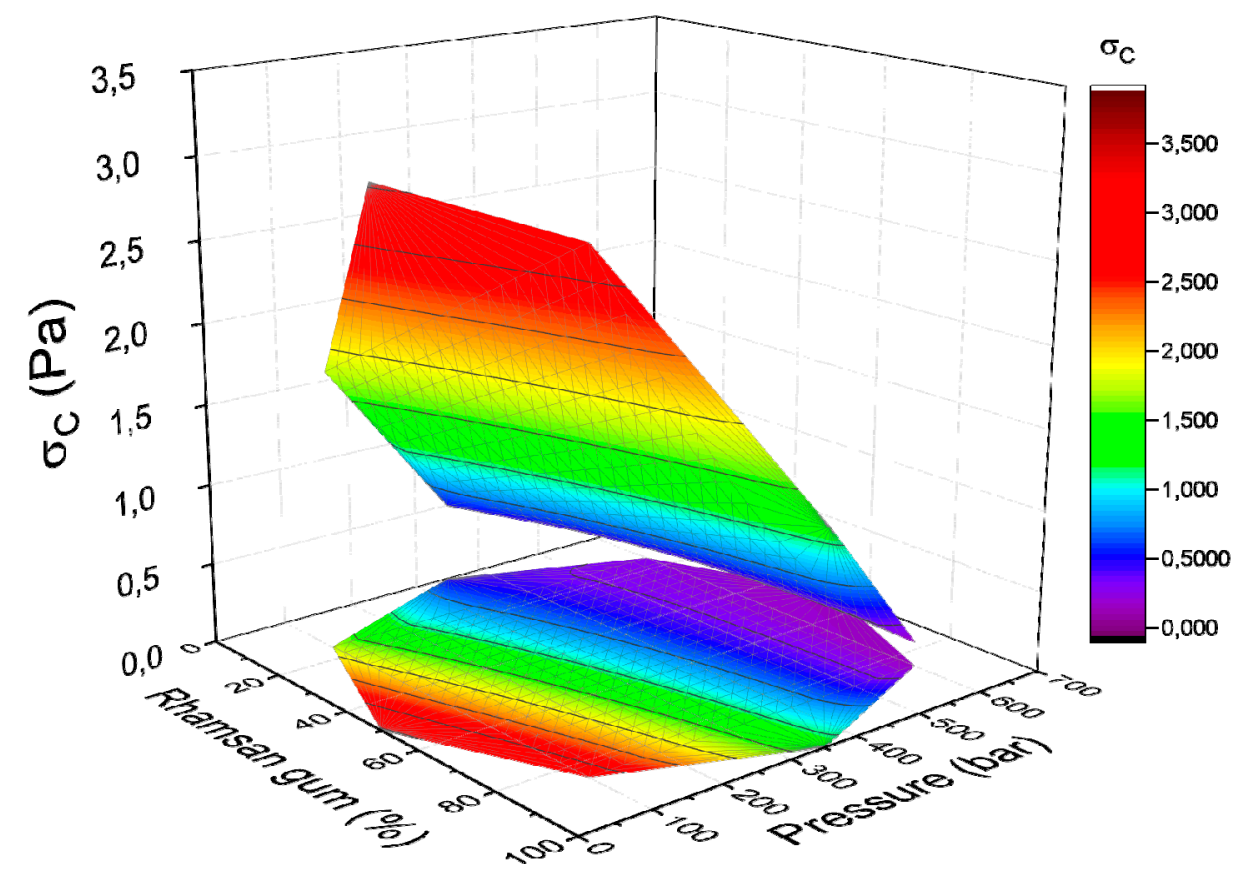

Figure 4. Three-dimensional response surface curve of critical shear stress $\left(\sigma_{\mathrm{c}}\right)$ for rhamsan gum $(\%)$ and homogenization pressure applied in a microfluidizer.

\subsection{Development of Thyme Oil-in-Water Emulsions}

Figure 5 shows the droplet size distribution (DSD) for the selected emulsion $(0.4 \mathrm{wt} \%$ rhamsan gum) processed at different homogenization pressures in the microfluidizer. The pre-emulsion (emulsion not homogenized in the microfluidizer) presents a bimodal distribution with a second peak centered at above $50 \mu \mathrm{m}$. In contrast, emulsions developed using the microfluidizer exhibited DSDs with only one peak centered below $10 \mu \mathrm{m}$. There was a clear reduction of droplet size using the microfluidizer for these thyme oil-in-water emulsions. In addition, a more marked decrease of droplet size was related to higher homogenization pressures. This fact has been reported before by other authors for emulsions without gums [27-29]. However, this reduction of droplet size observed for emulsions containing gums had not been reported yet. It is important to highlight that the incorporation of rhamsan gum before microfluidization was not a drawback for the reduction of droplet size. Furthermore, there was no existence of recoalescence due to overprocessing. This seems to indicate that continuous phase development (surfactant and rhamsan gum) protects the interface from a possible recoalescence effect.

Stress sweeps at $1 \mathrm{~Hz}$ for the selected emulsion processed at different homogenization pressures in the microfluidizer are shown in Figure 6. Emulsions developed with no microfluidizer and at 350 bars exhibited similar behaviors. Both systems show a plateau zone of elastic and loss moduli $\left(G^{\prime}, G^{\prime \prime}\right)$, which is the linear viscoelastic range (LVR), followed by an abrupt decrease with stress. $G^{\prime}$ is higher than $G^{\prime \prime}$ for the two abovementioned systems in the LVR. This is related to a gel behavior. However, the values of $G^{\prime}$ and $G^{\prime \prime}$ for the emulsions developed at 350 bars are lower than those for the emulsion not processed in the microfluidizer. This fact is not in concordance with the DSD observed in Figure 5 since lower values of diameters are usually related to higher values of $G^{\prime}$ and $G^{\prime \prime}$. In addition, the highest pressure applied (700 bar) to the selected emulsion provoked a decrease in both viscoelastic moduli, reaching similar values for $G^{\prime}$ and $G^{\prime \prime}$. This emulsion presented the lowest mean diameter 
in Figure 5. Hence, the use of the microfluidizer in this emulsion not only reduced the droplet size of the emulsion but also provoked a loss of microstructure developed by the gums. This can be also observed in the length of the plateau zone, i.e., there was a reduction of the LVR with the increase in the homogenization pressure. These conformation changes using microfluidization have been previously reported in xanthan gum solutions [13].

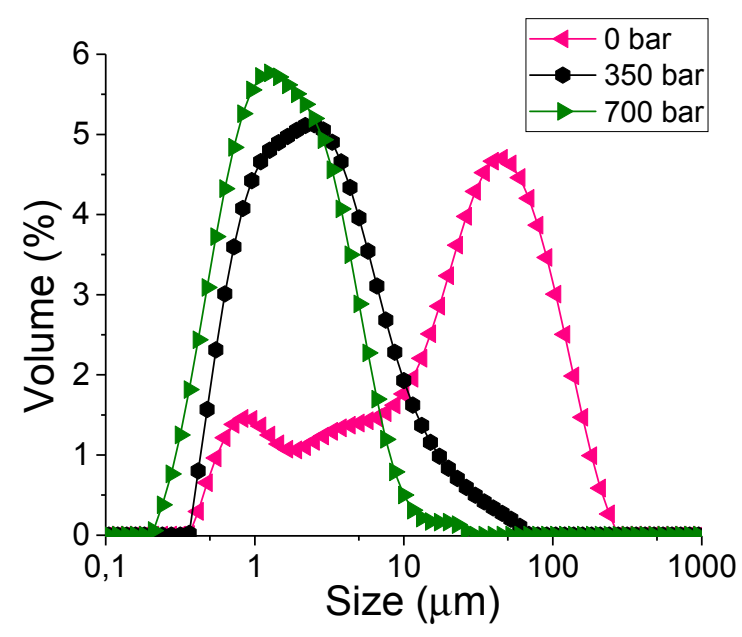

Figure 5. Droplet size distributions for thyme emulsions containing $0.4 \mathrm{wt} \%$ of rhamsan gum as a function of the homogenization pressure applied in the microfluidizer.

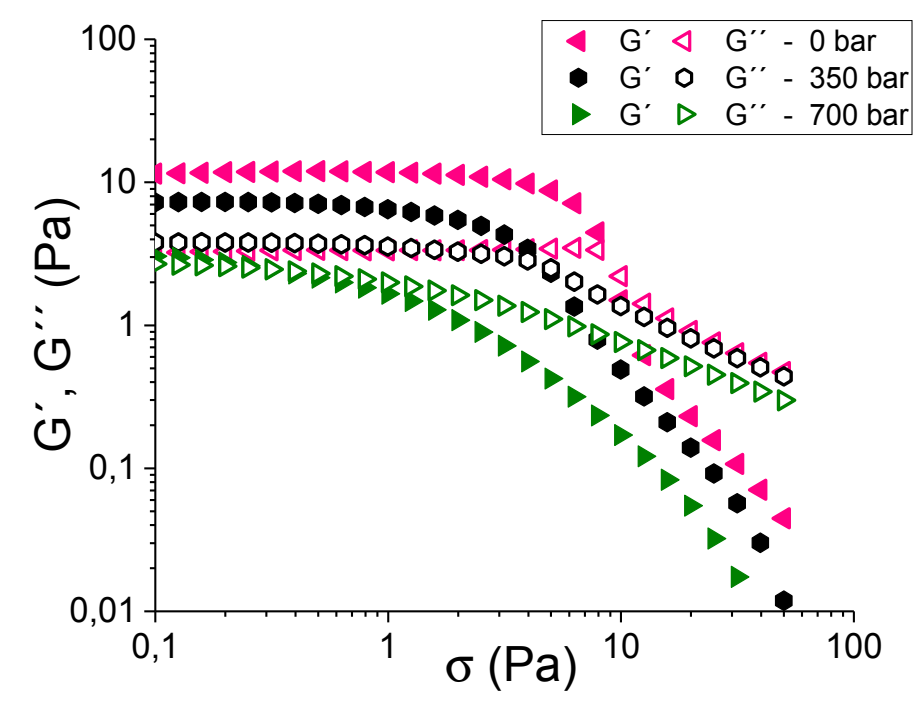

Figure 6. Stress sweeps for thyme emulsions studied containing $0.4 \mathrm{wt} \%$ of rhamsan gum as a function of the homogenization pressure applied in the microfluidizer.

Figure 7 shows the Turbiscan Stability Index (TSI) with aging time as a function of the homogenization pressure applied in the microfluidizer. It is important to mention that higher values of the TSI involve poorer physical stabilities [25,26]. All emulsions followed the same tendency in TSI values: a marked increase and subsequently a leveling off trend. However, the values were quite different. The emulsion processed at the highest homogenization pressure (700 bar) exhibited the lowest TSI values, proving its enhanced physical stability. Hence, although there is a loss of structuration using high homogenization pressures, the reduction of droplet size is the key parameter for the physical stability of these emulsions. 


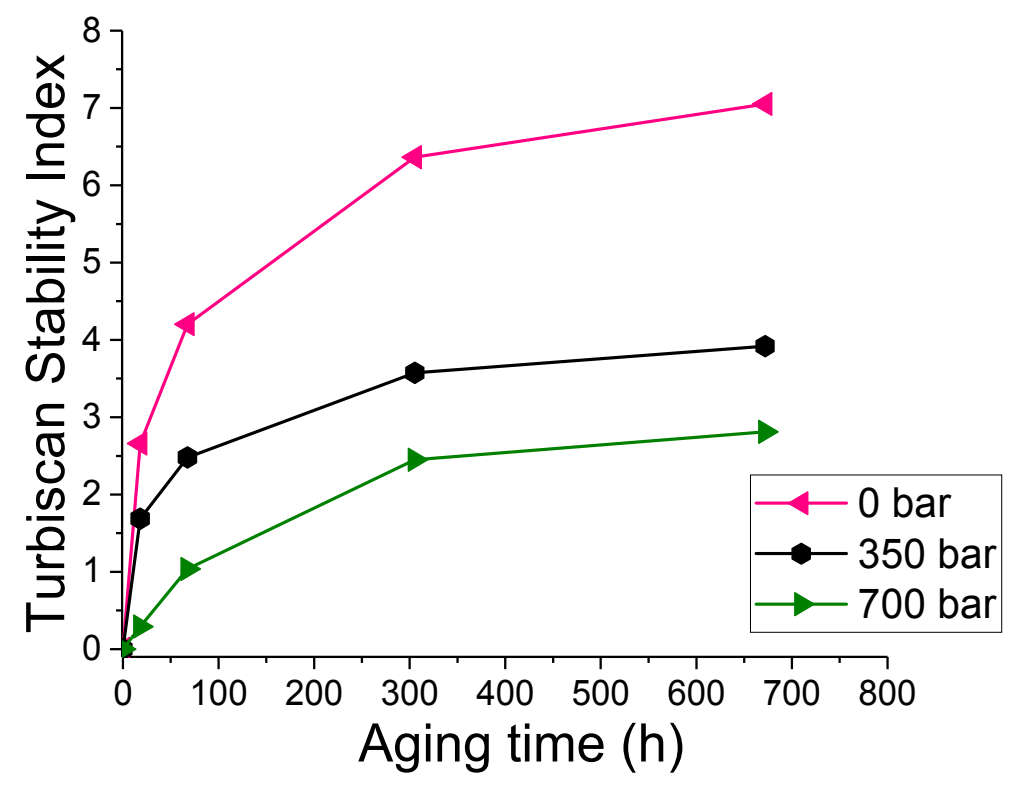

Figure 7. Turbiscan Stability Index parameter for thyme emulsions studied containing $0.4 \mathrm{wt} \%$ of rhamsan gum as a function of the homogenization pressure applied in the microfluidizer.

\section{Conclusions}

The influence of the gums' concentration ratio and homogenization pressure on the critical shear stress of dispersions containing a blend of welan and rhamsan gums was studied using design of experiments and surface response methodology. In order to rationally develop the formulation and processing conditions of biopolymer dispersions prepared with a microfluidizer, a quadratic model was required. An increase in the welan gum concentration and homogenization pressure provoked a decrease in critical shear stress values and weaker structures. An optimal combination of formulation and processing, with maximum critical shear stress, was obtained for $X_{1}=1.414$ and $X_{2}=0$. For this reason, the use of pure rhamsan gum and no pressure for the development of continuous phases was taken as a starting point for a further study of the influence of the homogenization pressure on the production of emulsions.

Thyme oil-in-water emulsions containing rhamsan gum were developed by using the microfluidization technique. The microfluidization technique has proved to be a powerful tool to reduce droplet size. However, this technique was also responsible for the decrease in the structuration grade of the studied emulsions. Hence, the break of gum structure was caused by the use of the microfluidizer, which was observed in the stress sweeps of the emulsions studied. This fact was pointed out not only by the lower viscoelastic functions but also by the smaller LVR. However, the reduction of droplet size led to the production of more stable emulsions, proved by the multiple light scattering technique. The emulsion processed at the highest homogenization pressure (700 bar) showed the greatest physical stability. This study can lay the foundation for the development of emulsions containing rhamsan gum via the microfluidization technique.

Author Contributions: Conceptualization, J.M. and M.d.C.A.; Methodology, L.A.T.-C.; Software, N.C.; Validation, N.C.; Formal Analysis, N.C.; Investigation, L.A.T.-C. and J.S.; Resources, J.M.; Data Curation, N.C.; Writing-Original Draft Preparation, J.S.; Writing-Review and Editing, L.A.T.-C. and J.S.; Visualization, L.A.T.-C.; Supervision, M.d.C.A.; Project Administration, J.M. and M.d.C.A.; Funding Acquisition, J.M. and M.d.C.A.

Acknowledgments: The financial support received (Project CTQ2015-70700-P) from the Spanish Ministerio de Economía y Competitividad and from the European Commission (FEDER Programme) is gratefully acknowledged.

Conflicts of Interest: The authors declare no conflict of interest. 


\section{References}

1. Robins, M.M.; Wilde, P.J. Colloids and emulsions. Science 2003, 1517-1524. [CrossRef]

2. Pérez-Mosqueda, L.M.; Ramírez, P.; Trujillo-Cayado, L.A.; Santos, J.; Muñoz, J. Development of eco-friendly submicron emulsions stabilized by a bio-derived gum. Colloids Surf. B Biointerfaces 2014, 123, 797-802. [CrossRef] [PubMed]

3. Qiu, C.; Zhao, M.; McClements, D.J. Improving the stability of wheat protein-stabilized emulsions: Effect of pectin and xanthan gum addition. Food Hydrocoll. 2015, 43, 377-387. [CrossRef]

4. Kang, K.S.; Pettitt, D.J. Xanthan, gellan, welan, and rhamsan. In Industrial Gums, 3rd ed.; Elsevier: Amsterdam, The Netherlands, 1993; pp. 341-397.

5. Trujillo-Cayado, L.A.; Alfaro, M.C.; Muñoz, J.; Raymundo, A.; Sousa, I. Development and rheological properties of ecological emulsions formulated with a biosolvent and two microbial polysaccharides. Colloids Surf. B Biointerfaces 2016, 141, 53-58. [CrossRef] [PubMed]

6. Trujillo-Cayado, L.A.; Alfaro, M.C.; Raymundo, A.; Sousa, I.; Muñoz, J. Rheological behavior of aqueous dispersions containing blends of rhamsan and welan polysaccharides with an eco-friendly surfactant. Colloids Surf. B Biointerfaces 2016, 145, 430-437. [CrossRef] [PubMed]

7. Xu, X.Y.; Dong, S.H.; Li, S.; Chen, X.Y.; Wu, D.; Xu, H. Statistical experimental design optimization of rhamsan gum production by Sphingomonas sp. CGMCC 6833. J. Microbiol. 2015, 53, 272-278. [CrossRef] [PubMed]

8. Hagiwara, A.; Imai, N.; Doi, Y.; Sano, M.; Tamano, S.; Omoto, T.; Asai, I.; Yasuhara, K.; Hayashi, S. Ninety-day oral toxicity study of rhamsan gum, a natural food thickener produced from Sphingomonas ATCC 31961, in Crl:CD(SD)IGS rats. J. Toxicol. Sci. 2010, 35, 493-501. [CrossRef] [PubMed]

9. Sanderson, G.R. Gellan gum. In Food Gels; Springer: New York, NY, USA, 1990; pp. 201-232.

10. Lee, L.; Norton, I.T. Comparing droplet breakup for a high-pressure valve homogeniser and a microfluidizer for the potential production of food-grade nanoemulsions. J. Food Eng. 2013, 114, 158-163. [CrossRef]

11. Jafari, S.M.; He, Y.; Bhandari, B. Optimization of nano-emulsions production by microfluidization. Eur. Food Res. Technol. 2007, 225, 733-741. [CrossRef]

12. Santos, J.; Trujillo-Cayado, L.A.; Calero, N.; Alfaro, M.C.; Muñoz, J. Development of eco-friendly emulsions produced by microfluidization technique. J. Ind. Eng. Chem. 2016, 36, 90-95. [CrossRef]

13. Lagoueyte, N.; Paquin, P. Effects of microfluidization on the functional properties of xanthan gum. Food Hydrocoll. 1998, 12, 365-371. [CrossRef]

14. Xu, L.; Xu, G.; Liu, T.; Chen, Y.; Gong, H. The comparison of rheological properties of aqueous welan gum and xanthan gum solutions. Carbohydr. Polym. 2013, 92, 516-522. [CrossRef] [PubMed]

15. Ruberto, G.; Baratta, M.T.; Deans, S.G.; Dorman, H.J.D. Antioxidant and antimicrobial activity of Foeniculum vulgare and Crithmum maritimum essential oils. Planta Med. 2000, 66, 687-693. [CrossRef] [PubMed]

16. Satou, T.; Hayakawa, M.; Goto, Y.; Masuo, Y.; Koike, K. Anxiolytic-like effects of essential oil from Thymus vulgaris was increased during stress. Flavour Fragr. J. 2018, 33, 191-195. [CrossRef]

17. Dobetsberger, C.; Buchbauer, G. Actions of essential oils on the central nervous system: An updated review. Flavour Fragr. J. 2011, 26, 300-316. [CrossRef]

18. Kwiatkowski, P.; Mnichowska-Polanowska, M.; Pruss, A.; Masiuk, H.; Dzięcioł, M.; Giedrys-Kalemba, S.; Sienkiewicz, M. The effect of fennel essential oil in combination with antibiotics on Staphylococcus aureus strains isolated from carriers. Burns 2017, 43, 1544-1551. [CrossRef] [PubMed]

19. Ryu, V.; McClements, D.J.; Corradini, M.G.; McLandsborough, L. Effect of ripening inhibitor type on formation, stability, and antimicrobial activity of thyme oil nanoemulsion. Food Chem. 2018, 245, 104-111. [CrossRef] [PubMed]

20. Santos, J.; Calero, N.; Trujillo-Cayado, L.A.; Muñoz, J. Development and characterisation of a continuous phase based on a fumed silica and a green surfactant with emulsion applications. Colloids Surf. A Physicochem. Eng. Asp. 2018, 555, 351-357. [CrossRef]

21. Zasypkin, D.V.; Braudo, E.E.; Tolstoguzov, V.B. Multicomponent biopolymer gels. Food Hydrocoll. 1997, 11, 159-170. [CrossRef]

22. Kennedy, J.F.; Woods, J.R.; Harding, S.E.; Hill, S.E.; Mitchell, J.R. Biopolymer Mixtures. Bioseparation 1998, 7, 64. [CrossRef] 
23. Mengual, O.; Meunier, G.; Cayré, I.; Puech, K.; Snabre, P. TURBISCAN MA 2000: Multiple light scattering measurement for concentrated emulsion and suspension instability analysis. Talanta 1999, 50, 445-456. [CrossRef]

24. Santos, J.; Trujillo, L.A.; Calero, N.; Alfaro, M.C.; Munoz, J. Physical Characterization of a Commercial Suspoemulsion as a Reference for the Development of Suspoemulsions. Chem. Eng. Technol. 2013, 36, 1883-1890. [CrossRef]

25. Santos, J.; Calero, N.; Trujillo-Cayado, L.A.; Garcia, M.C.; Muñoz, J. Assessing differences between Ostwald ripening and coalescence by rheology, laser diffraction and multiple light scattering. Colloids Surf. B Biointerfaces 2017, 159, 405-411. [CrossRef] [PubMed]

26. Trujillo-Cayado, L.A.; Alfaro, M.C.; Muñoz, J. Effects of ethoxylated fatty acid alkanolamide concentration and processing on D-limonene emulsions. Colloids Surf. A Physicochem. Eng. Asp. 2018, 536, 198-203. [CrossRef]

27. Trujillo-Cayado, L.A.; Santos, J.; Ramírez, P.; Alfaro, M.C.; Muñoz, J. Strategy for the development and characterization of environmental friendly emulsions by microfluidization technique. J. Clean. Prod. 2018, 178, 723-730. [CrossRef]

28. Martin-Piñero, M.J.; Ramirez, P.; Muñoz, J.; Alfaro, M.C. Development of rosemary essential oil nanoemulsions using a wheat biomass-derived surfactant. Colloids Surf. B Biointerfaces 2019, 173, 486-492. [CrossRef] [PubMed]

29. Jo, Y.-J.; Kwon, Y.-J. Characterization of $\beta$-carotene nanoemulsions prepared by microfluidization technique. Food Sci. Biotechnol. 2014, 23, 107-113. [CrossRef]

(c) 2019 by the authors. Licensee MDPI, Basel, Switzerland. This article is an open access article distributed under the terms and conditions of the Creative Commons Attribution (CC BY) license (http://creativecommons.org/licenses/by/4.0/). 